\title{
APPROXIMATING QUASISTATIONARY DISTRIBUTIONS OF BIRTH-DEATH PROCESSES
}

\author{
DAMIAN CLANCY, ${ }^{*}$ University of Liverpool
}

\begin{abstract}
For a sequence of finite state space birth-death processes, each having a single absorbing state, we show that, under certain conditions, as the size of the state space tends to infinity, the quasistationary distributions converge to the stationary distribution of a limiting infinite state space birth-death process. This generalizes a result of Keilson and Ramaswamy by allowing birth and death rates to depend upon the size of the state space. We give sufficient conditions under which the convergence result of Keilson and Ramaswamy remains valid. The generalization allows us to apply our convergence result to examples from population biology: a Pearl-Verhulst logistic population growth model and the susceptible-infective-susceptible (SIS) model for infectious spread. The limit distributions obtained suggest new finite-population approximations to the quasistationary distributions of these models, obtained by the method of cumulant closure. The new approximations are found to be both simple in form and accurate.
\end{abstract}

Keywords: Moment closure; cumulant closure; SIS epidemic model; logistic population growth

2010 Mathematics Subject Classification: Primary 60J28

Secondary 60J80; 92D25; 92D30

\section{Introduction}

Consider a birth-death process $\left\{X^{(k)}(t): t \geq 0\right\}$ on the state space $S^{(k)}=\{0,1,2, \ldots, k+1\}$. Suppose that there is one absorbing state at $k+1$, with the remaining states forming a single communicating class $C^{(k)}$. Then the long-term behaviour of the process prior to eventual absorption is described by the limiting conditional distribution $\boldsymbol{q}^{(k)}=\left\{q_{i}^{(k)}: i \in C^{(k)}\right\}$, where

$$
q_{i}^{(k)}=\lim _{t \rightarrow \infty} \operatorname{Pr}\left(X^{(k)}(t)=i \mid X^{(k)}(t) \in C^{(k)}\right) .
$$

This distribution is known to be unique, and is also the unique quasistationary distribution, in the sense that if $\operatorname{Pr}\left(X^{(k)}(0)=i\right)=q_{i}^{(k)}$ for $i \in C^{(k)}$ then $\operatorname{Pr}\left(X^{(k)}(t)=i \mid X^{(k)}(t) \in C^{(k)}\right)=q_{i}^{(k)}$ for all $t>0$ (see [6]). Henceforth, we shall follow common usage in referring to $\boldsymbol{q}^{(k)}$ as the quasistationary distribution.

In general, no explicit closed form can be found for the elements $q_{i}^{(k)}$ of the quasistationary distribution, and so in specific applications one may seek to approximate. One general technique is to approximate the quasistationary distribution by the stationary distribution of a limiting diffusion process; see, e.g. [20]. Another general approach, which we discuss in more detail in Section 4, is cumulant closure (or, equivalently, moment closure): having first chosen some standard distributional form with which to approximate the quasistationary distribution, one

Received 14 June 2011; revision received 2 May 2012.

* Postal address: Department of Mathematical Sciences, University of Liverpool L69 7ZL, UK.

Email address: d.clancy@liv.ac.uk 
then seeks to evaluate the first few cumulants of the approximating distribution; see, e.g. [12]. In the specific case of the widely studied susceptible-infective-susceptible (SIS) infection model, the recent monograph by Nåsell [16] (see also [17]) gives a very thorough asymptotic analysis of the quasistationary distribution in the large population limit, while a review of various other approximations from the literature appears in Clancy and Mendy [3]. A more general PearlVerhulst logistic population growth model, which includes the SIS model as a special case, has been similarly analysed by Nåsell [13], [14], [15].

In a different vein, Keilson and Ramaswamy [8] showed that, under certain conditions, the quasistationary distributions of a sequence of birth-death processes $X^{(k)}$ converge as $k \rightarrow \infty$ to the stationary distribution of a limiting birth-death process on the infinite, irreducible state space $S=\{0,1, \ldots\}$, implying that the probabilities $q_{i}^{(k)}$ may be approximated using the stationary distribution of the limiting process. In [8], the birth and death rates from any given state $i$ remain fixed as $k \rightarrow \infty$. In Section 2 we extend this result to allow transition rates to vary with $k$. The extension is motivated by examples such as those of Section 3, where we apply our convergence result to the Pearl-Verhulst logistic population growth model and the SIS infection model. The rigorous convergence results of Section 3 suggest new cumulant-closure approximations to the quasistationary distributions of the Pearl-Verhulst and SIS models, which we develop in Section 4. In Section 5 we present some numerical work, demonstrating that our new cumulant-closure approximations can improve upon previously known approximations. Finally, Section 6 contains some concluding discussion.

All numerical work was carried out using MATLAB ${ }^{\circledR}$ on a desktop PC.

\section{Convergence of quasistationary distributions}

For $k=1,2, \ldots$, suppose that $\left\{X^{(k)}(t): t \geq 0\right\}$ is a continuous-time birth-death process on the state space $S^{(k)}=\{0,1,2, \ldots, k+1\}$, with $S^{(k)}$ consisting of a communicating class $C^{(k)}=\{0,1,2, \ldots, k\}$ and an absorbing state $k+1$. Equivalently, denoting by $\lambda_{i}^{(k)}$ and $\mu_{i}^{(k)}$ the birth and death rates, respectively, from state $i$, then we have $\lambda_{0}^{(k)}, \lambda_{1}^{(k)}, \ldots, \lambda_{k}^{(k)}>0, \lambda_{k+1}^{(k)}=0$, $\mu_{0}^{(k)}=0, \mu_{1}^{(k)}, \mu_{2}^{(k)}, \ldots, \mu_{k}^{(k)}>0$, and $\mu_{k+1}^{(k)}=0$. Denote by $\boldsymbol{q}^{(k)}=\left(q_{0}^{(k)}, q_{1}^{(k)}, \ldots, q_{k}^{(k)}\right)$ the unique quasistationary distribution of $X^{(k)}$.

Following Keilson and Ramaswamy [8], consider two modified versions of the process $X^{(k)}$. First, consider the process reflected at $k$ (equivalently, set $\lambda_{k}^{(k)}=0$ ), and denote by $\rho^{(k)}$ the stationary distribution of this reflected process. Next, consider the process re-started from state 0 whenever state $k+1$ is reached, and denote by $\boldsymbol{v}^{(k)}$ the stationary distribution of this re-started process. Keilson and Ramaswamy [8] showed that

$$
\boldsymbol{v}^{(k)} \prec_{\text {st }} \boldsymbol{q}^{(\boldsymbol{k})} \prec_{\mathrm{st}} \boldsymbol{\rho}^{(k)},
$$

where ' $\prec_{\text {st }}$ ' denotes the usual stochastic ordering of distributions. An alternative proof of the relations in (2.1) appears in Clancy and Pollett [4], where an explicit algebraic form for the stationary distribution of the re-started process is given. Note that the reflected process may equally well be regarded as being re-started from state $k$ whenever state $k+1$ is reached, so that this process also falls within the framework of re-started processes studied by Clancy and Pollett [4], following Ferrari et al. [7].

Defining $\pi_{0}^{(k)}=1$ and

$$
\pi_{i}^{(k)}=\frac{\lambda_{0}^{(k)} \lambda_{1}^{(k)} \cdots \lambda_{i-1}^{(k)}}{\mu_{1}^{(k)} \mu_{2}^{(k)} \cdots \mu_{i}^{(k)}} \quad \text { for } i=1,2, \ldots, k,
$$


then it is well known that the elements of $\boldsymbol{\rho}^{(k)}$ are given by

$$
\rho_{i}^{(k)}=\frac{\pi_{i}^{(k)}}{\sum_{j=0}^{k} \pi_{j}^{(k)}} \quad \text { for } i=0,1, \ldots, k .
$$

On the other hand, from Clancy and Pollett [4], the elements of $\boldsymbol{v}^{(k)}$ are given by

$$
v_{i}^{(k)}=\frac{\pi_{i}^{(k)} \sum_{j=i}^{k}\left(\lambda_{j}^{(k)} \pi_{j}^{(k)}\right)^{-1}}{\sum_{j=0}^{k}\left(\lambda_{j}^{(k)} \pi_{j}^{(k)}\right)^{-1} \sum_{r=0}^{j} \pi_{r}^{(k)}} \quad \text { for } i=0,1, \ldots, k .
$$

Suppose now that $\{X(t): t \geq 0\}$ is some continuous-time birth-death process on the irreducible state space $S=\{0,1,2, \ldots\}$, so that, denoting by $\lambda_{i}$ and $\mu_{i}$ the birth and death rates, respectively, from state $i$, we have $\lambda_{0}, \lambda_{1}, \ldots>0, \mu_{0}=0, \mu_{1}, \mu_{2}, \ldots>0$. With $\pi_{0}=1$, and $\pi_{i}$ defined for $i=1,2, \ldots$ by (2.2) with the superscript $(k)$ omitted, define

$$
m_{i}=\frac{\pi_{i}}{\sum_{j=0}^{\infty} \pi_{j}} \quad \text { for } i=0,1, \ldots
$$

We will assume that $\sum_{j=0}^{\infty} \pi_{j}<\infty$, so that $\boldsymbol{m}=\left(m_{0}, m_{1}, \ldots\right)$ is a proper distribution. If also $\sum_{j=0}^{\infty}\left(\lambda_{j} \pi_{j}\right)^{-1}=\infty$ then the process $X$ is nonexplosive with stationary distribution $\boldsymbol{m}$. In what follows, we do not require that $\sum_{j=0}^{\infty}\left(\lambda_{j} \pi_{j}\right)^{-1}=\infty$; if this condition is not satisfied then the interpretation of $\boldsymbol{m}$ becomes a little more complicated, in that the process $X$ is now explosive and $\boldsymbol{m}$ is not the stationary distribution of the minimal process, but rather the stationary distribution of a nonminimal process having birth and death rates $\lambda_{i}$ and $\mu_{i}$.

The proof of our main result, Theorem 2.1 below, is based upon ideas from Keilson and Ramaswamy [8], but, on the one hand, we are able to proceed more directly using the explicit expressions (2.4) for the elements of $\boldsymbol{v}^{(k)}$ (in addition to the explicit expressions (2.3) for the elements of $\left.\boldsymbol{\rho}^{(k)}\right)$, while, on the other hand, the dependence of $\lambda_{i}^{(k)}$ and $\mu_{i}^{(k)}$ upon $k$ means that a little more care is needed in dealing with various summations.

Theorem 2.1. Suppose that $\sum_{j=1}^{k}\left|\pi_{j}^{(k)}-\pi_{j}\right| \rightarrow 0$ as $k \rightarrow \infty$. Furthermore, defining

$$
a_{i}^{(k)}=\frac{\left(\lambda_{i}^{(k)} \pi_{i}^{(k)}\right)^{-1}}{\sum_{j=0}^{k}\left(\lambda_{j}^{(k)} \pi_{j}^{(k)}\right)^{-1}} \quad \text { for } i=0,1, \ldots, k,
$$

suppose that $a_{i}^{(k)} \rightarrow 0$ as $k \rightarrow \infty$ for each $i=0,1, \ldots$

Then $\boldsymbol{q}^{(k)} \rightarrow \boldsymbol{m}$ elementwise as $k \rightarrow \infty$.

Proof. Due to the relations in (2.1), it suffices to show that $\boldsymbol{v}^{(k)} \rightarrow \boldsymbol{m}$ and $\boldsymbol{\rho}^{(k)} \rightarrow \boldsymbol{m}$.

First, consider convergence of $\boldsymbol{\rho}^{(k)}$ to $\boldsymbol{m}$. From (2.3) and (2.5),

$$
\left(\rho_{0}^{(k)}\right)^{-1}-\left(m_{0}\right)^{-1}=\sum_{j=0}^{k} \pi_{j}^{(k)}-\sum_{j=0}^{\infty} \pi_{j} \leq \sum_{j=1}^{k}\left|\pi_{j}^{(k)}-\pi_{j}\right|-\sum_{j=k+1}^{\infty} \pi_{j},
$$

and so (recalling that $\sum_{j=0}^{\infty} \pi_{j}<\infty$ ) we have $\rho_{0}^{(k)} \rightarrow m_{0}$ as $k \rightarrow \infty$. Now, since $\rho_{i}^{(k)}=$ $\pi_{i}^{(k)} \rho_{0}^{(k)}$ for $i=1,2, \ldots, k$ and $m_{i}=\pi_{i} m_{0}$ for $i=1,2, \ldots$, it is immediate that $\rho_{i}^{(k)} \rightarrow m_{i}$ as $k \rightarrow \infty$ for each $i$. 
Next consider the convergence of $\boldsymbol{v}^{(k)}$ to $\boldsymbol{m}$. From (2.4) we have

$$
\left(v_{0}^{(k)}\right)^{-1}=\sum_{i=0}^{k} a_{i}^{(k)} \sum_{j=0}^{i} \pi_{j}^{(k)},
$$

corresponding to Equation (3.25) of [8]. Noting that $\sum_{i=0}^{k} a_{i}^{(k)}=1$, then

$$
\begin{aligned}
\left(v_{0}^{(k)}\right)^{-1}-\left(m_{0}\right)^{-1} & =\sum_{i=0}^{k} a_{i}^{(k)} \sum_{j=0}^{i} \pi_{j}^{(k)}-\sum_{j=0}^{\infty} \pi_{j} \\
& =\sum_{j=0}^{k} \sum_{i=j}^{k} a_{i}^{(k)}\left(\pi_{j}^{(k)}-\pi_{j}\right)-\sum_{i=0}^{k} a_{i}^{(k)} \sum_{j=i+1}^{\infty} \pi_{j} .
\end{aligned}
$$

For any fixed $K$, we have, for $k>K$,

$$
\begin{aligned}
\left|\left(v_{0}^{(k)}\right)^{-1}-\left(m_{0}\right)^{-1}\right| & \leq \sum_{j=1}^{k}\left|\pi_{j}^{(k)}-\pi_{j}\right|+\sum_{i=0}^{K} a_{i}^{(k)} \sum_{j=i+1}^{\infty} \pi_{j}+\sum_{i=K+1}^{k} a_{i}^{(k)} \sum_{j=i+1}^{\infty} \pi_{j} \\
& \leq \sum_{j=1}^{k}\left|\pi_{j}^{(k)}-\pi_{j}\right|+\sum_{i=0}^{K} a_{i}^{(k)} \sum_{j=0}^{\infty} \pi_{j}+\sum_{j=K+2}^{\infty} \pi_{j} .
\end{aligned}
$$

Given $\varepsilon>0$, fix $K$ sufficiently large that $\sum_{j=K+2}^{\infty} \pi_{j}<\varepsilon / 3$. Then take $k_{0}>K$ sufficiently large that, for $k \geq k_{0}$, we have $a_{i}^{(k)}<\varepsilon\left(3(K+1) \sum_{j=0}^{\infty} \pi_{j}\right)^{-1}$ for $i=0,1, \ldots, K$ and also $\sum_{j=1}^{k}\left|\pi_{j}^{(k)}-\pi_{j}\right|<\varepsilon / 3$. Then, for $k \geq k_{0}$,

$$
\left|\left(v_{0}^{(k)}\right)^{-1}-\left(m_{0}\right)^{-1}\right| \leq \varepsilon .
$$

Thus, $v_{0}^{(k)} \rightarrow m_{0}$ as $k \rightarrow \infty$.

Now consider $v_{i}^{(k)}$ for $i \geq 1$. From (2.4), we have, for $i=0,1,2, \ldots, k$,

$$
v_{i}^{(k)}=\pi_{i}^{(k)} v_{0}^{(k)} \sum_{j=i}^{k} a_{j}^{(k)}=\pi_{i}^{(k)} v_{0}^{(k)}\left(1-\sum_{j=0}^{i-1} a_{j}^{(k)}\right) .
$$

For fixed $i \geq 1$, as $k \rightarrow \infty$, we have $\pi_{i}^{(k)} \rightarrow \pi_{i}, 0 \leq v_{0}^{(k)} \leq 1$, and $a_{j}^{(k)} \rightarrow 0$ for $j=$ $0,1, \ldots, i-1$. Hence, $v_{i}^{(k)}-\pi_{i}^{(k)} v_{0}^{(k)} \rightarrow 0$ as $k \rightarrow \infty$, and so $v_{i}^{(k)} \rightarrow \pi_{i} m_{0}=m_{i}$.

From the relations in (2.1), it follows that $\boldsymbol{q}^{(\boldsymbol{k})} \rightarrow \boldsymbol{m}$ elementwise as $k \rightarrow \infty$, as required.

Note that if we set $\lambda_{i}^{(k)}=\lambda_{i}$ and $\mu_{i}^{(k)}=\mu_{i}$ for $i=0,1, \ldots, k$, then the conditions of Theorem 2.1 reduce to precisely the condition required by Keilson and Ramaswamy [8] that $\sum_{j=0}^{\infty}\left(\lambda_{j} \pi_{j}\right)^{-1}=\infty$.

Remark. For any finite $k$, we have shown that the quasistationary distribution $\boldsymbol{q}^{(k)}$ may be approximated by the limiting stationary distribution $\boldsymbol{m}$; alternatively, we may choose to approximate $\boldsymbol{q}^{(k)}$ by $\boldsymbol{v}^{(k)}$ or $\boldsymbol{\rho}^{(k)}$. More generally, when the process $X^{(k)}(t)$ hits state $k+1$, we could re-start according to any (fixed) distribution $\boldsymbol{p}^{(k)}$ on $\{0,1,2, \ldots, k\}$, and denote by $\Phi\left(\boldsymbol{p}^{(k)}\right)$ the stationary distribution of this re-started process. Clancy and Pollett [4] showed that $\boldsymbol{v}^{(k)} \prec_{\operatorname{lr}} \Phi\left(\boldsymbol{p}^{(k)}\right) \prec_{\operatorname{lr}} \boldsymbol{\rho}^{(k)}$ for any $\boldsymbol{p}^{(k)}$, where ' $\prec_{\operatorname{lr}}$ ' denotes the likelihood ratio ordering, 
which implies the usual stochastic ordering. It follows that, for any sequence of distributions $\boldsymbol{p}^{(k)}$ on $\{0,1,2, \ldots, k\}$, we have $\Phi\left(\boldsymbol{p}^{(k)}\right) \rightarrow \boldsymbol{m}$ componentwise as $k \rightarrow \infty$, so that, for large $k$, $\Phi\left(\boldsymbol{p}^{(k)}\right)$ may be reasonably used as an approximation to $\boldsymbol{q}^{(k)}$. The fact that in many practical applications $\Phi\left(\boldsymbol{p}^{(k)}\right)$ provides a reasonable approximation to $\boldsymbol{q}^{(k)}$ for any re-start distribution $\boldsymbol{p}^{(k)}$ has previously been observed by Barbour and Pollett [2].

\section{Applications}

\subsection{The Pearl-Verhulst logistic population model}

Nåsell [13] formulated a stochastic version of the Pearl-Verhulst model for population growth as follows. Take $\tilde{X}^{(N)}(t)$ to be a birth and death process on the state space $\{0,1, \ldots, N\}$ with birth and death rates

$$
\begin{aligned}
& \tilde{\lambda}_{i}^{(N)}= \begin{cases}\lambda i\left(1-\alpha_{1} \frac{i}{N}\right) & \text { for } i=0,1, \ldots, N-1, \\
0 & \text { for } i=N,\end{cases} \\
& \tilde{\mu}_{i}^{(N)}=\mu i\left(1+\alpha_{2} \frac{i}{N}\right) \text { for } i=0,1, \ldots, N,
\end{aligned}
$$

where $\lambda, \mu>0,0 \leq \alpha_{1} \leq 1$, and $\alpha_{2} \geq 0$. This process is ultimately absorbed at state 0 , so in order to apply Theorem 2.1 , we set $k+1=N$ and define the process $X^{(k)}(t)=k+1-\tilde{X}^{(N)}(t)$. The process $X^{(k)}$ is ultimately absorbed at state $k+1$ and has rates

$$
\begin{aligned}
& \lambda_{i}^{(k)}=\mu(k+1-i)\left(1+\alpha_{2} \frac{k+1-i}{k+1}\right) \\
& \mu_{i}^{(k)}= \begin{cases}0 & \text { for } i=0,1, \ldots, k, \\
\lambda(k+1-i)\left(1-\alpha_{1} \frac{k+1-i}{k+1}\right) & \text { for } i=1,2, \ldots, k .\end{cases}
\end{aligned}
$$

Hence, for $i=1,2, \ldots, k$, we have

$$
\pi_{i}^{(k)}=\frac{(\mu / \lambda)^{i}(k+1)}{k+1-i} \prod_{j=0}^{i-1}\left(\frac{1+\alpha_{2}(k+1-j) /(k+1)}{1-\alpha_{1}(k-j) /(k+1)}\right),
$$

so that, for fixed $i$, as $k \rightarrow \infty$,

$$
\pi_{i}^{(k)} \rightarrow\left(\frac{\mu\left(1+\alpha_{2}\right)}{\lambda\left(1-\alpha_{1}\right)}\right)^{i}=\pi_{i}
$$

We require the parameters to satisfy $\mu\left(1+\alpha_{2}\right)<\lambda\left(1-\alpha_{1}\right)$ in order that $\sum_{i=0}^{\infty} \pi_{i}<\infty$. Note that the case $\alpha_{1}=1$ will be discussed separately in Section 3.2 below.

To apply Theorem 2.1 , we must first check that $\sum_{j=1}^{k}\left|\pi_{j}^{(k)}-\pi_{j}\right| \rightarrow 0$ as $k \rightarrow \infty$. Setting $\theta=\mu\left(1+\alpha_{2}\right) / \lambda\left(1-\alpha_{1}\right)$ and

$$
B_{k i}=\frac{1-\left(\alpha_{2} /\left(1+\alpha_{2}\right)\right)((i-1) /(k+1))}{1+\left(\alpha_{1} /\left(1-\alpha_{1}\right)\right)(i /(k+1))},
$$


then, for $i=1,2, \ldots, k$, we have $\pi_{i}=\theta^{i}$ and

$$
\theta^{i} B_{k i}^{i} \leq \pi_{i}^{(k)} \leq \theta^{i}\left(1+\frac{i}{k+1-i}\right)
$$

Since $0<B_{k i} \leq 1$, then $1-B_{k i}^{i} \leq i\left(1-B_{k i}\right)$, and so

$$
\begin{aligned}
\sum_{i=1}^{k}\left|\pi_{i}^{(k)}-\pi_{i}\right| & \\
\leq & \sum_{i=1}^{k} \theta^{i}\left(\frac{i}{k+1-i}+1-B_{k i}^{i}\right) \\
\leq & \sum_{i=1}^{k} \theta^{i}\left(\frac{i}{k+1-i}+i\left(1-B_{k i}\right)\right) \\
= & \sum_{i=1}^{k} \theta^{i} i\left(\frac{1}{k+1-i}\right. \\
\leq & \frac{r_{k}}{k+1-r_{k}} \sum_{i=1}^{r_{k}} \theta^{i}+\sum_{i=r_{k}+1}^{k} i \theta^{i} \\
& +\frac{1}{k+1}\left(\frac{\alpha_{1}}{1-\alpha_{1}} \sum_{i=1}^{\infty} i^{2} \theta^{i}+\frac{\alpha_{2}}{1+\alpha_{2}} \sum_{i=1}^{\infty} i(i-1) \theta^{i}\right),
\end{aligned}
$$

where $r_{k}$ is any sequence of integers with $1 \leq r_{k} \leq k$. Since all four series in the above expression are convergent, then choosing the $r_{k}$ such that $r_{k} \rightarrow \infty$ and $r_{k} / k \rightarrow 0$ as $k \rightarrow \infty$ ensures that all the terms on the right-hand side of the inequality converge to 0 as $k \rightarrow \infty$, as required.

To check the condition that $a_{i}^{(k)} \rightarrow 0$ as $k \rightarrow \infty$, note that

$$
\frac{\lambda_{i}^{(k)} \pi_{i}^{(k)}}{k+1}=\mu\left(\frac{\mu}{\lambda}\right)^{i}\left(1+\alpha_{2} \frac{k+1-i}{k+1}\right) \prod_{j=0}^{i-1}\left(\frac{1+\alpha_{2}(k+1-j) /(k+1)}{1-\alpha_{1}(k-j) /(k+1)}\right),
$$

so that $\lambda_{i}^{(k)} \pi_{i}^{(k)} /(k+1) \rightarrow \mu\left(1+\alpha_{2}\right) \theta^{i}$ as $k \rightarrow \infty$, and also

$$
\left(\frac{\lambda_{i}^{(k)} \pi_{i}^{(k)}}{k+1}\right)^{-1} \geq \frac{1}{\mu\left(1+\alpha_{2}\right) \theta^{i}} \text {. }
$$

Hence, for $\theta<1$,

$$
a_{i}^{(k)} \leq \frac{\mu\left(1+\alpha_{2}\right)\left(\lambda_{i}^{(k)} \pi_{i}^{(k)} /(k+1)\right)^{-1}}{\sum_{j=0}^{k}(1 / \theta)^{j}} \rightarrow 0 \quad \text { as } k \rightarrow \infty .
$$

The conditions of Theorem 2.1 are all satisfied, and so, as $k \rightarrow \infty$, the quasistationary distribution of the process $X^{(k)}$ converges to a geometric distribution on $\{0,1,2, \ldots\}$. In terms of the 
Pearl-Verhulst logistic model $\tilde{X}^{(N)}$, with quasistationary distribution $\left(\tilde{q}_{1}^{(N)}, \tilde{q}_{2}^{(N)}, \ldots, \tilde{q}_{N}^{(N)}\right)$, then, for large $N$, we have

$$
\tilde{q}_{i}^{(N)} \approx(1-\theta) \theta^{N-i} \text { for } i=1,2, \ldots, N,
$$

provided $\theta<1$.

To clarify the interpretation of the condition $\theta<1$, consider the deterministic process $\tilde{x}(t)$ corresponding to the proportion $\tilde{X}^{(N)}(t) / N$ in the limit as $N \rightarrow \infty$. Then $\tilde{x}(t) \in[0,1]$ and

$$
\frac{\mathrm{d} \tilde{x}}{\mathrm{~d} t}=\tilde{x}\left(\lambda-\mu-\left(\alpha_{1} \lambda+\alpha_{2} \mu\right) \tilde{x}\right) \quad \text { for } 0 \leq \tilde{x}<1 .
$$

Thus, $\tilde{x}(t)$ has an equilibrium point at $\tilde{x}=0$, which is stable for $\lambda \leq \mu$. Defining $a=$ $(\lambda-\mu) /\left(\alpha_{1} \lambda+\alpha_{2} \mu\right)$ then, provided $0<a<1$, there is a second equilibrium point at $\tilde{x}=a$, and this point is stable for $0<a<1$ (note that $a>0$ is equivalent to $\lambda>\mu$ ). In the case $a>1, \tilde{x}(t)$ reaches the boundary at $\tilde{x}=1$ within finite time, provided $\tilde{x}(0)>0$. The general form of the birth rates $\tilde{\lambda}_{i}^{(N)}=\lambda i\left(1-\alpha_{1} i / N\right)$ does not reduce to 0 at the boundary $i=N$, and instead the model imposes $\tilde{\lambda}_{N}^{(N)}=0$ as a separate condition. Consequently, $\mathrm{d} \tilde{x} / \mathrm{d} t$ has a discontinuity at $\tilde{x}=1$. Provided the process does not tend to spend much time near the boundary at $\tilde{X}^{(N)}=N$, the somewhat artificial nature of the boundary condition need not be an issue. In the case $a>1$, however, the process does tend to spend time near this boundary. The condition $a>1$ is equivalent to $\theta<1$. Thus, although the case $\theta<1$ is of some mathematical interest, the more biologically relevant case is when $\theta>1$.

\subsection{The Pearl-Verhulst model in the case $\alpha_{1}=1$}

In the case $\alpha_{1}=1$ the limit result above does not apply. On the other hand, the general form for $\tilde{\lambda}_{i}^{(N)}$ does now reduce to 0 at $i=N$, and we have $a<1$, so that this is a more biologically realistic case. The model with $\alpha_{1}=1$ was studied by Norden [18].

A proper limit distribution may be obtained by scaling the parameter $\lambda$ as $\lambda=N \bar{\lambda}$ for some fixed $\bar{\lambda}>0$. We thus obtain

$$
\pi_{i}^{(k)}=\frac{(\mu / \bar{\lambda})^{i}}{i !} \frac{k+1}{k-i+1} \prod_{j=0}^{i-1}\left(1+\alpha_{2} \frac{k+1-j}{k+1}\right) \rightarrow \frac{\phi^{i}}{i !}=\pi_{i} \quad \text { as } k \rightarrow \infty,
$$

where $\phi=\mu\left(1+\alpha_{2}\right) / \bar{\lambda}$. We have $\sum_{i=1}^{\infty} \pi_{i}<\infty$ and

$$
\frac{\phi^{i}}{i !}\left(1-\frac{\alpha_{2}}{1+\alpha_{2}} \frac{i-1}{k+1}\right)^{i}<\pi_{i}^{(k)}<\frac{\phi^{i}}{i !}\left(1+\frac{i}{k+1-i}\right) .
$$

Hence,

$$
\begin{aligned}
\sum_{i=1}^{k}\left|\pi_{i}^{(k)}-\pi_{i}\right| & \leq \sum_{i=1}^{k} \frac{\phi^{i}}{i !}\left(\frac{i}{k+1-i}+1-\left(1-\frac{\alpha_{2}}{1+\alpha_{2}} \frac{i-1}{k+1}\right)^{i}\right) \\
& \leq \sum_{i=1}^{k} \frac{\phi^{i}}{i !}\left(\frac{i}{k+1-i}+i \frac{\alpha_{2}}{1+\alpha_{2}} \frac{i-1}{k+1}\right) \\
& \leq \phi \sum_{i=0}^{k-1} \frac{\phi^{i}}{i !} \frac{1}{k-i}+\frac{1}{k+1} \frac{\alpha_{2}}{1+\alpha_{2}} \phi^{2} \mathrm{e}^{\phi}
\end{aligned}
$$


Taking $r_{k}$ to be a sequence of integers with $1 \leq r_{k} \leq k$ such that, as $k \rightarrow \infty$, we have $r_{k} \rightarrow \infty$ and $r_{k} / k \rightarrow 0$, then

$$
\sum_{i=0}^{k-1} \frac{\phi^{i}}{i !} \frac{1}{k-i} \leq \frac{1}{k-r_{k}} \sum_{i=0}^{r_{k}} \frac{\phi^{i}}{i !}+\sum_{i=r_{k}+1}^{k-1} \frac{\phi^{i}}{i !} \leq \frac{\mathrm{e}^{\phi}}{k-r_{k}}+\sum_{i=r_{k}+1}^{\infty} \frac{\phi^{i}}{i !} \rightarrow 0 \quad \text { as } k \rightarrow \infty,
$$

and so $\sum_{i=1}^{k}\left|\pi_{i}^{(k)}-\pi_{i}\right| \rightarrow 0$ as $k \rightarrow \infty$.

Finally,

$$
\frac{\lambda_{i}^{(k)} \pi_{i}^{(k)}}{k+1}=\frac{\mu(\mu / \bar{\lambda})^{i}}{i !} \prod_{j=0}^{i}\left(1+\alpha_{2} \frac{k+1-j}{k+1}\right)
$$

so that $\lambda_{i}^{(k)} \pi_{i}^{(k)} /(k+1) \rightarrow \mu\left(1+\alpha_{2}\right) \phi^{i} / i !$ as $k \rightarrow \infty$ and

$$
\left(\frac{\lambda_{i}^{(k)} \pi_{i}^{(k)}}{k+1}\right)^{-1} \geq \frac{1}{\mu\left(1+\alpha_{2}\right)} \frac{i !}{\phi^{i}},
$$

from which it is immediate that $a_{i}^{(k)} \rightarrow 0$ as $k \rightarrow \infty$ for each fixed $i$.

The quasistationary distribution of $X^{(k)}(t)$ thus converges to a Poisson distribution of mean $\phi$. Hence, for large $N$, the quasistationary probabilities of the Pearl-Verhulst model $\tilde{X}^{(N)}$ in the case $\alpha_{1}=1$, with scaled birth rate parameter $\lambda=N \bar{\lambda}$, satisfy

$$
\tilde{q}_{i}^{(N)} \approx \frac{\phi^{N-i}}{(N-i) !} \mathrm{e}^{-\phi} \quad \text { for } i=1,2, \ldots, N .
$$

\subsection{The SIS model for endemic infection}

In the case $\alpha_{1}=1, \alpha_{2}=0$, the Pearl-Verhulst model reduces to the SIS infection model of Weiss and Dishon [21]. In this context, $\tilde{X}^{(N)}$ represents the number of infective individuals in a closed population of size $N$, with $X^{(k)}$ representing the number of susceptible individuals. For large $N$, the quasistationary probabilities, expressed in terms of the number of infectives, satisfy

$$
\tilde{q}_{i}^{(N)} \approx \frac{\left(N / R_{0}\right)^{N-i}}{(N-i) !} \mathrm{e}^{-N / R_{0}} \quad \text { for } i=1,2, \ldots, N,
$$

where $R_{0}=N / \phi=\lambda / \mu$ is known as the basic reproduction number of the infection process.

Note that we are here using a limiting distribution on $\{1,2, \ldots\}$ to approximate a distribution on $\{1,2, \ldots, N\}$, and so our limiting probabilities (3.3) do not sum to 1 . We can improve the approximation by normalizing to obtain

$$
\tilde{q}_{i}^{(N)} \approx\left(\frac{R_{0}}{N}\right)^{i} \frac{1}{(N-i) !} / \sum_{j=1}^{N}\left(\frac{R_{0}}{N}\right)^{j} \frac{1}{(N-j) !} \quad \text { for } i=1,2, \ldots, N .
$$

Approximation (3.4) is precisely the approximation suggested by Kryscio and Lefèvre [9] for the nonendemic case $R_{0}<1$. Our limit result, in contrast, requires $R_{0}=N / \phi$ with $\phi$ fixed as $N \rightarrow \infty$, so that we expect approximation (3.3) to work well only for $R_{0} \gg 1$.

Whether for the SIS model, the Pearl-Verhulst model, or, more generally, our limiting result, suggests that $\boldsymbol{q}^{(k)} \approx \boldsymbol{m}$, but we would expect to improve the approximation by normalizing. That is, with $\boldsymbol{m}^{(k)}=\left(\sum_{i=0}^{k} m_{i}\right)^{-1} \boldsymbol{m}$, an improved approximation is $\boldsymbol{q}^{(k)} \approx \boldsymbol{m}^{(k)}$. We shall not pursue this further, since we seek approximations of simple form. We note that in the case of the SIS model, Clancy and Pollett [4] showed that $\boldsymbol{m}^{(k)} \prec_{\text {st }} \boldsymbol{q}^{(k)}$. 


\section{Cumulant closure approximations}

Cumulant closure is a general technique that may be used to approximate the quasistationary distribution of a birth-death process as follows. First, write down the Kolmogorov forward equations for the state probabilities. Next, condition upon the event $\left\{X^{(k)}(t) \in C^{(k)}\right\}$ to obtain a set of ordinary differential equations satisfied by the conditional state probabilities $\operatorname{Pr}\left(X^{(k)}(t)=i \mid X^{(k)}(t) \in C^{(k)}\right)$ for $i \in C^{(k)}$. By summing over the nonabsorbing states one can then obtain a partial differential equation satisfied by the conditional moment generating function $M(\theta, t)=\mathrm{E}\left[\exp \left(\theta X^{(k)}(t)\right) \mid X^{(k)}(t) \in C^{(k)}\right]$. Taking logs leads to a partial differential equation satisfied by the cumulant generating function $K(\theta, t)=\ln M(\theta, t)$. Expanding as a power series in $\theta$ yields expressions for the time derivatives of the cumulants $k_{1}(t), k_{2}(t), \ldots$ of the distribution of the state of the process at time $t$ conditional upon nonabsorption. We set these derivatives equal to 0 and solve to find the equilibrium values $k_{1}, k_{2}, \ldots$ The expression for the derivative of the cumulant of any particular order will in general contain terms of higher order, and so before we can solve the equations we need to somehow close the system. We do this by assuming an appropriate distributional form for the quasistationary distribution of interest, allowing all higher-order cumulants to be expressed in terms of the first few cumulants. Full details for the case of the SIS infection model may be found in Clancy and Mendy [3]; see Nåsell [14], [15] for the case of the Pearl-Verhulst model.

A key step in the cumulant closure method is the choice of a specific distributional form with which to approximate the quasistationary distribution of interest. Most commonly, the normal distribution is used, so that cumulants of order 3 and above vanish, leaving two equations to be solved for the two unknowns $k_{1}$ and $k_{2}$. To improve upon the normal approximation, one can try a variety of distributional forms and see which leads to the best approximation. Thus, for the SIS model in the case $R_{0}>1$, Clancy and Mendy [3] used normal, lognormal, binomial, negative binomial, and beta-binomial distributions to approximate the quasistationary distribution of the number of infectives, and concluded that of these the beta-binomial distribution gave the best approximation. Whereas Clancy and Mendy [3] simply experimented with many different distributions in a somewhat arbitrary manner, in Sections 4.1-4.3 we use the results obtained in Section 3 to provide guidance in the choice of an appropriate approximating distribution.

\subsection{The Pearl-Verhulst model}

Starting from the Kolmogorov forward equations of the process $\tilde{X}^{(N)}(t)$, Nåsell [14] derived expressions for the time derivatives of the first three cumulants. In the case $\alpha_{1} \neq 1$, however, there are terms missing from the expressions given by Nåsell [14]. In particular, the first cumulant differential equation should be

$$
\begin{aligned}
\dot{k}_{1}= & \frac{\lambda-\mu}{a N}\left(\left(a N-k_{1}(t)\right) k_{1}(t)-k_{2}(t)\right)+\mu\left(1+\frac{\alpha_{2}}{N}\right) \tilde{q}_{1}^{(N)}(t) k_{1}(t) \\
& -\lambda\left(1-\alpha_{1}\right) N \tilde{q}_{N}^{(N)}(t),
\end{aligned}
$$

where $\tilde{q}_{i}^{(N)}(t)=\operatorname{Pr}\left(\tilde{X}^{(N)}(t)=i \mid \tilde{X}^{(N)}(t) \neq 0\right)$. The final term, involving $\tilde{q}_{N}^{(N)}(t)$, is omitted from the equations quoted by Nåsell [14]. This term arises because of the discontinuity in the form of the birth rates $\tilde{\lambda}_{i}^{(N)}$ at the boundary $i=N$.

Our limit result suggests that, provided $\theta<1$, the distribution of $N-\tilde{X}^{(N)}$ in quasistationarity may be approximated by a geometric distribution supported on $\{0,1,2, \ldots\}$. Assuming the geometric distributional form, but without assuming the parameter value of 
the limit theorem, implies that

$$
\begin{gathered}
k_{2}=\left(N-k_{1}\right)\left(N-k_{1}+1\right), \\
\tilde{q}_{N}^{(N)}=\frac{1}{N-k_{1}+1},
\end{gathered}
$$

and it remains to find $k_{1}$. Since $\theta<1$ (equivalently, $a>1$ ), we may treat $\tilde{q}_{1}^{(N)}$ as negligible. Substituting (4.2) and (4.3) into (4.1) and neglecting the term in $\tilde{q}_{1}^{(N)}$, then, close to equilibrium,

$$
\dot{k}_{1} \approx \frac{\lambda-\mu}{a N\left(N-k_{1}+1\right)} f\left(k_{1}\right)
$$

where, for $x \in \mathbb{R}$, we define

$$
f(x)=2 x^{3}-((4+a) N+3) x^{2}+(N+1)((3+a) N+1) x-N(N+1)^{2}-\frac{a-1}{1-\theta} N^{2} .
$$

Any stable equilibrium point must satisfy $f\left(k_{1}\right)=0$ and $f^{\prime}\left(k_{1}\right)<0$. This is only possible if the equation $f(x)=0$ has three real roots, with the middle root providing the stable equilibrium point $k_{1}$. With $\theta<1$, so that $a>1$, it is clear that $f(x)<0$ for $x \leq 0$ and that $f(N)<0$, so that $k_{1}$ must be the larger of the two roots within $(0, N)$, provided the equation $f(x)=0$ has a root within this range.

The limiting result (3.1) implies that, for large $N$, provided $\theta<1$,

$$
k_{1} \approx N-\frac{\theta}{1-\theta}
$$

Asymptotically, as $N \rightarrow \infty$, we find that one of the roots of $f(x)=0$ has leading-order terms in agreement with this, while the other two roots do not; in fact, the relevant root satisfies

$$
k_{1}=N-\frac{\theta}{1-\theta}-\frac{\theta}{(1-\theta)^{2}}\left(1-\frac{1}{a-1}\right) \frac{1}{N}+O\left(\frac{1}{N^{2}}\right) .
$$

For numerical work, we shall evaluate $k_{1}$ by solving the equation $f(x)=0$ numerically using the MATLAB fsolve function to search for solutions close to $x=N$.

\subsection{The Pearl-Verhulst model in the case $\alpha_{1}=1$}

When $\alpha_{1}=1$, in order that we can neglect terms in $\tilde{q}_{1}^{(N)}$, we require $\lambda \gg \mu$, so that $0 \ll a<1$. With this assumption, (4.1) in equilibrium reduces to

$$
\left(a N-k_{1}\right) k_{1}-k_{2}=0 .
$$

For this case, we obtained a Poisson limit for the process $X^{(k)}$, which would imply that the cumulants for $\tilde{X}^{(N)}$ satisfy $k_{2}=N-k_{1}$. With this substitution, (4.4) yields

$$
k_{1}=\frac{1}{2}\left(a N+1+\sqrt{\left.\left((\sqrt{N}-1)^{2}-(1-a) N\right)\left((\sqrt{N}+1)^{2}-(1-a) N\right)\right)},\right.
$$

where we take the positive square root to ensure a stable equilibrium point. For a real solution, we require $a \geq 1-(1-(1 / \sqrt{N}))^{2}$. 


\subsection{The SIS model for endemic infection}

With $\alpha_{1}=1, \alpha_{2}=0$, and $R_{0}=\lambda / \mu,(4.5)$ may be written as

$$
k_{1}=\frac{1}{2}\left(N\left(1-\frac{1}{R_{0}}\right)+1+\sqrt{\left((\sqrt{N}-1)^{2}-\frac{N}{R_{0}}\right)\left((\sqrt{N}+1)^{2}-\frac{N}{R_{0}}\right)},\right.
$$

and, for real roots, we require $R_{0} \geq(1-(1 / \sqrt{N}))^{-2}$. Note that $k_{1}=N\left(1-1 / R_{0}\right)-$ $1 /\left(R_{0}-1\right)+O(1 / N)$, which is in agreement with the asymptotic expression for $k_{1}$ given by Clancy and Mendy [3].

The Poisson moment closure approximation is remarkably simple, in that we have assumed a one-parameter distributional form and, thus, only needed to use a single cumulant equation. Previous cumulant closure approximations have most often fitted two-parameter distributional families, which immediately suggests that we may be able to improve upon our Poisson approximation using a negative binomial distribution. Clancy and Mendy [3] considered, inter alia, a negative binomial approximation for the quasistationary distribution of the number of infectives in the SIS model. Here we consider a negative binomial approximation for the number of susceptibles.

This distributional assumption implies the cumulant relationship

$$
k_{3}=k_{2}-\frac{2 k_{2}^{2}}{N-k_{1}} .
$$

When $R_{0} \gg 1$, so that terms involving $\tilde{q}_{1}^{(N)}$ may be neglected, the first two cumulant equilibrium equations are

$$
\begin{gathered}
\left(N\left(1-\frac{1}{R_{0}}\right)-k_{1}\right) k_{1}-k_{2}=0 \\
\left(N\left(1+\frac{1}{R_{0}}\right)-k_{1}\right) k_{1}+\left(2 N\left(1-\frac{1}{R_{0}}\right)-1\right) k_{2}-4 k_{1} k_{2}-2 k_{3}=0 .
\end{gathered}
$$

Substituting for $k_{2}$ and $k_{3}$ from (4.6) and (4.7) into (4.8) gives a cubic equation for $k_{1}$ which can be straightforwardly solved to yield solutions $k_{1}=0$ or

$$
\begin{aligned}
k_{1}=N & \left(\left(1-\frac{1}{R_{0}}\right)\left(1-\frac{N}{2\left(N+N R_{0}-R_{0}\right)}\right)\right. \\
& \left. \pm \frac{\sqrt{\left((\sqrt{N}-\sqrt{2})^{2}-N / R_{0}\right)\left((\sqrt{N}+\sqrt{2})^{2}-N / R_{0}\right)}}{2\left(N+N R_{0}-R_{0}\right)}\right) .
\end{aligned}
$$

For real roots, we require $R_{0} \geq(1-\sqrt{2 / N})^{-2}$. To leading order in $N$, taking the positive square root in the above expression gives $k_{1}=\left(1-1 / R_{0}\right) N+O(1)$, which is in agreement with previous moment closure approximations and, therefore, provides the relevant solution. We can now obtain $k_{2}$ immediately from (4.7).

\section{Performance of the approximations}

We follow Clancy and Mendy [3] in measuring the performance of our approximations in terms of the total variation distance restricted to the support of the true quasistationary distribution. That is, if $\boldsymbol{q}$ denotes the quasistationary distribution of interest supported on 
$\{1,2, \ldots, N\}$, and $\hat{\boldsymbol{q}}$ denotes an approximating distribution, the distance between the two is defined to be

$$
d(\boldsymbol{q}, \hat{\boldsymbol{q}})=\frac{1}{2} \sum_{i=1}^{N}\left|q_{i}-\hat{q}_{i}\right| .
$$

For all of the approximations $\hat{\boldsymbol{q}}$ considered below, it may be possible to improve the numerical accuracy by re-normalizing so that the probabilities sum to 1 , as discussed at the end of Section 3.3, but we do not pursue this here.

\subsection{The Pearl-Verhulst model}

Figure 1 shows the relative performance of the geometric limit result (3.1) and the geometric cumulant closure approximation with $\alpha_{1}=0.5, \alpha_{2}=0$, and $N=50$, over a range of values of the ratio $R_{0}=\lambda / \mu$. With these values, the condition $\theta<1$ reduces to $R_{0}>2$. We see that the cumulant closure approximation gives a noticeable improvement upon the limit result. For larger values of $N$, a similar picture is seen, with both approximations becoming more accurate as $N$ increases. So far as the parameters $\alpha_{1}$ and $\alpha_{2}$ are concerned, in general, the condition $\theta<1$ may be expressed as $R_{0}>\left(1+\alpha_{2}\right) /\left(1-\alpha_{1}\right)$, so that increasing either of $\alpha_{1}$ or $\alpha_{2}$ reduces the range of $R_{0}$ values over which our limit result can be applied. Numerical work indicates that both of our approximations become more accurate as $\alpha_{1}$ and $\alpha_{2}$ decrease.

\subsection{The Pearl-Verhulst model with $\alpha_{1}=1$}

Figure 2 shows the relative performance of the Poisson limit result (3.2) and the Poisson cumulant closure approximation with $\alpha_{1}=1, \alpha_{2}=0.2$, and $N=50$, over a range of values of $R_{0}$. For these parameter values, the condition for a real solution to (4.5) is $R_{0} \geq 1.428$ (approximately). Again, the cumulant closure approximation gives a noticeable improvement upon the limiting result. The limit result here approximates the distribution of $X^{(k)}$ by a Poisson distribution of mean $\phi=N\left(1+\alpha_{2}\right) / R_{0}$, and since $X^{(k)} \in\{0,1, \ldots, N-1\}$, we would only

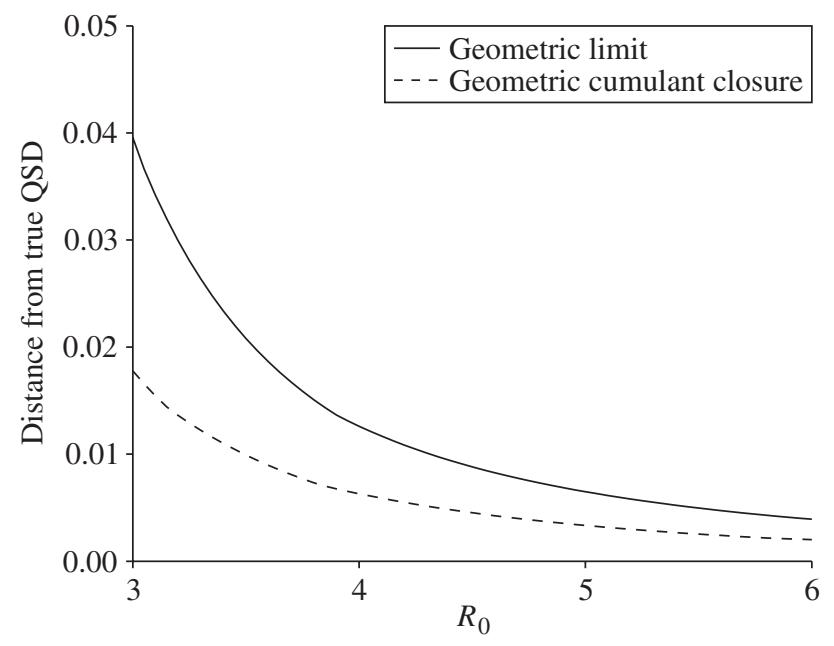

FIGURE 1: Distance between approximating distributions and the true quasistationary distribution of the Pearl-Verhulst logistic model as $R_{0}=\lambda / \mu$ varies, with $\alpha_{1}=0.5, \alpha_{2}=0$, and $N=50$. 


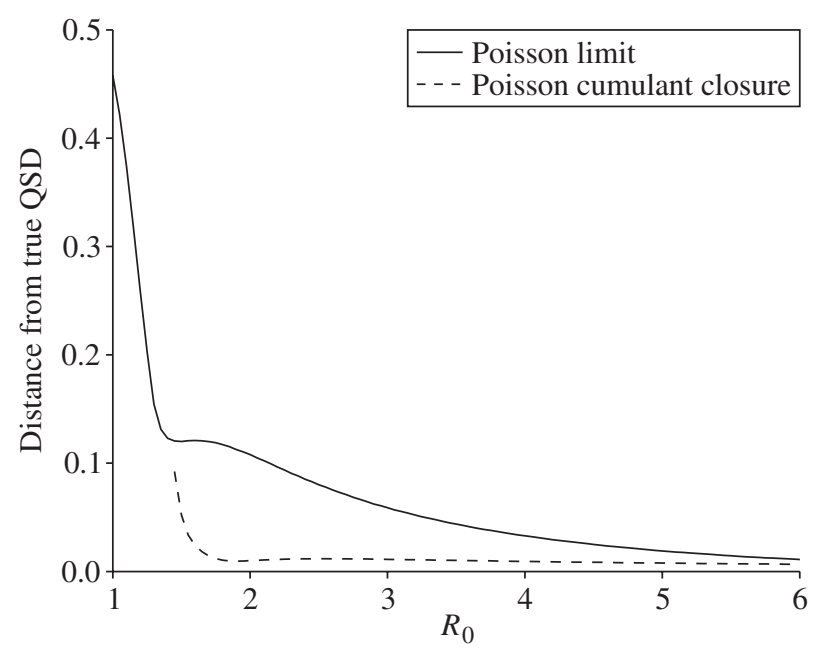

FiguRE 2: Distance between approximating distributions and the true quasistationary distribution of the Pearl-Verhulst logistic model as $R_{0}=\lambda / \mu$ varies, with $\alpha_{1}=1, \alpha_{2}=0.2$, and $N=50$.

expect a good approximation provided $1+\alpha_{2} \ll R_{0}$. In fact, we find, as before, that the smaller the value of $\alpha_{2}$, the better both of our approximations perform. For very small $\alpha_{2}$, increasing $N$ improves the accuracy of both approximations, as one might expect. For larger values, increasing $N$ while keeping $\alpha_{2}$ and $R_{0}$ fixed seems to have very little effect upon the accuracy of the cumulant closure approximation, while the Poisson limit approximation actually becomes less accurate as $N$ increases. Note that the limiting regime of Section 3.2 requires that $R_{0}$ be proportional to $N$, rather than remaining fixed as $N$ increases.

\subsection{The SIS model for endemic infection}

By far the most widely studied case is the SIS infection model, with $\alpha_{1}=1$ and $\alpha_{2}=0$. Many authors have derived approximations to the quasistationary distribution of this model; a review of the various approximations appears in Clancy and Mendy [3]. The best approximation for moderate $R_{0}$ values was found by Clancy and Mendy to be that of Ovaskainen [19], given by

$$
\tilde{q}_{i}^{\mathrm{Ov}}=K N\left(1-\frac{N}{(N-1) R_{0}}\right) \mathrm{e}^{-N / R_{0}}\left(1-\left(\frac{N}{(N-1) R_{0}}\right)^{i}\right) \frac{\left(N / R_{0}\right)^{N-i}}{i(N-i) !}
$$

for $i=1,2, \ldots, N-1$, where

$$
K=\max \left\{1, \frac{2(N-1)^{2} R_{0}^{2}}{N\left((N-1) R_{0}-N\right)^{2}}\right\},
$$

with the probability $\tilde{q}_{N}^{\text {Ov }}$ being given by a slightly modified version of (5.1). This approximation was criticised in [3] as performing poorly for large values of $R_{0}$. However, closer inspection reveals that it is only the probability $\tilde{q}_{N}^{(N)}$ which is poorly approximated, and that if we simply use (5.1) with $i=N$ instead of the modified formula for $\tilde{q}_{N}^{\text {Ov }}$ suggested in [19] then there is no problem for large $R_{0}$ values. Consequently, we shall compare our own approximations only with that of [19], which itself outperforms all other approximations considered in [3]. 


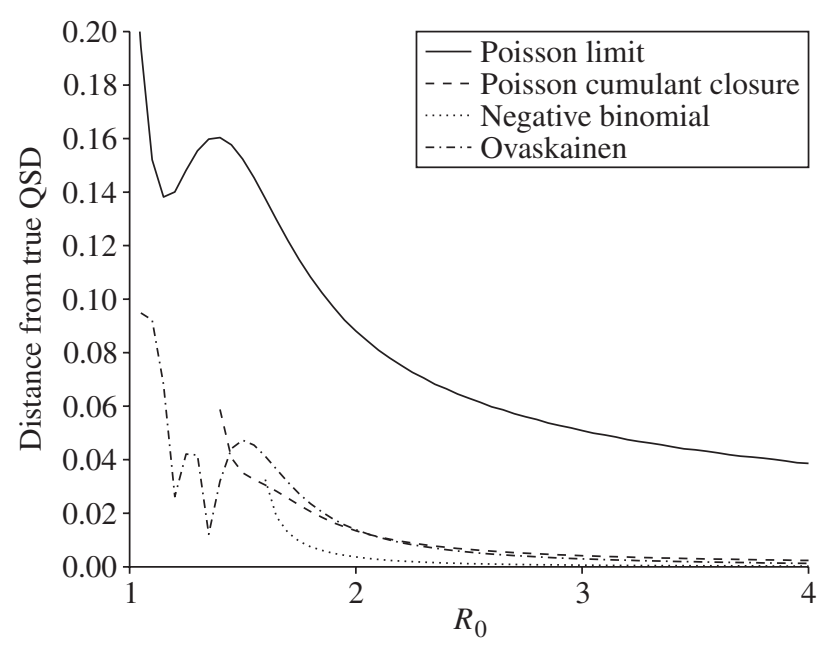

FIGURE 3: Distance between approximating distributions and the true quasistationary distribution of the SIS infection model as $R_{0}=\lambda / \mu$ varies, with $N=50$.

We take $N=50$, so that the condition for the Poisson cumulant closure method to yield a real root is $R_{0} \geq 1.357$ (approximately), while the corresponding condition for the negative binomial cumulant closure method is $R_{0} \geq 1.5625$. Figure 3 shows that the Poisson cumulant closure approximation performs almost as well as Ovaskainen's approximation, while being of rather simpler form. The approximation obtained by cumulant closure assuming a negative binomial distribution for the number of susceptibles in quasistationarity outperforms even Ovaskainen's approximation.

For larger values of $N$, a similar picture is seen, with all of the approximations becoming more accurate as $N$ increases, in line with our finding of Section 5.2 for sufficiently small values of $\alpha_{2}$.

\section{Discussion}

The quasistationary distribution of the SIS infection model has been much studied in the literature, as, to a lesser extent, have those of more general logistic population models. The convergence result of Keilson and Ramaswamy [8] is not directly applicable to such models, but by extending their result we have been able to establish convergence for the quasistationary distributions of these processes, and, hence, to develop new and more accurate approximations.

On the face of it, our limiting regime does not appear interesting, since in the applications we have considered the probability mass is concentrated close to an upper boundary which in the limit goes to infinity. In the case $\alpha_{1}=1$, this amounts to the fact that we have $R_{0} \rightarrow \infty$, when it would seem more reasonable to hold $R_{0}$ fixed as $N \rightarrow \infty$. However, what is more important for applications is whether the limiting result provides a reasonably accurate approximation for finite $N$. We see from Figures 2 and 3 that we can indeed obtain reasonably accurate approximations (of very simple form) for moderate $R_{0}$ values for which the probability mass of the quasistationary distribution is substantially spread across the state space. At the expense of slightly greater complexity, the cumulant closure approximations suggested by our limit result can give very good agreement with the true quasistationary distribution for moderate $R_{0}$ values. 
For the SIS model, our convergence result firstly suggests that it may be more productive to consider the distribution of the number of susceptible individuals, rather than focusing (as previous authors have done) on the number of infective individuals. Focusing upon the number of susceptibles has previously enjoyed some success when applied to susceptible-infectiveremoved (SIR) infection models, with the object of interest now being not a quasistationary distribution but rather the distribution of the total number of individuals remaining uninfected at the end of an outbreak of infection. Daniels [5] established a Poisson limit theorem for this quantity in the case of a major outbreak, with more general and extensive results having since been obtained by Ball and Barbour [1] and Lefèvre and Utev [10], [11]. Secondly, our convergence result suggests appropriate distributional forms to consider as approximations to the quasistationary distribution. In terms of a balance between simplicity and accuracy, arguably the most satisfactory approximation is obtained by applying the cumulant closure method assuming a Poisson-distributed number of susceptibles. Although the negative binomial cumulant closure approximation and the approximation of Ovaskainen [19] are both more accurate (for sufficiently large $R_{0}$ ), the Poisson approximation retains almost as much accuracy with significantly greater simplicity.

A finite state space birth-death process of the form we have considered will almost surely be absorbed within finite time. The quasistationary distribution is always well defined for such a process, but is only of practical interest if the time until absorption is long. In terms of our Pearl-Verhulst model, this is equivalent to the condition $a \gg 0$, and allows us to treat $\tilde{q}_{1}^{(N)}$ as negligible in deriving our cumulant closure approximations. If the time until absorption is only moderately long, it may be that the process does have time to settle to its quasistationary distribution before absorption occurs, but that $\tilde{q}_{1}^{(N)}$ is not small enough to be neglected. Deriving satisfactory approximations to the quasistationary distribution then becomes a challenging task, and even for the SIS model no really satisfactory solution has been found in this parameter region (where $R_{0}$ is only slightly above 1 ).

We noted in Section 3 that our Poisson limit distribution for the SIS model becomes, on normalization, the approximation suggested by Kryscio and Lefèvre [9] for $R_{0}<1$, whereas our unnormalized version is applicable for $R_{0} \gg 1$. In the case $R_{0} \gg 1$, normalization makes very little difference, so we expect the normalized Poisson approximation (3.4) to work well for both $R_{0}<1$ and $R_{0} \gg 1$, suggesting that this approximation may work well across the whole range of $R_{0}$ values. In fact, the approximation does indeed seem reasonably accurate for all $R_{0}$ values, but becomes least accurate when $R_{0}$ is slightly above 1 .

\section{References}

[1] Ball, F. G. And Barbour, A. D. (1990). Poisson approximation for some epidemic models. J. Appl. Prob. 27, 479-490.

[2] Barbour, A. D. And Pollett, P. K. (2010). Total variation approximation for quasi-stationary distributions. J. Appl. Prob. 47, 934-946.

[3] Clancy, D. And Mendy, S. T. (2011). Approximating the quasi-stationary distribution of the SIS model for endemic infection. Methodology Comput. Appl. Prob. 13, 603-618.

[4] Clancy, D. and Pollett, P. K. (2003). A note on quasi-stationary distributions of birth-death processes and the SIS logistic epidemic. J. Appl. Prob. 40, 821-825.

[5] Daniels, H. E. (1967). The distribution of the total size of an epidemic. In Proc. 5th Berkeley Symp. Math. Statist. Prob., Vol. 4, University of California Press, Berkeley, CA, pp. 281-293.

[6] Darroch, J. N. And Seneta, E. (1967). On quasi-stationary distributions in absorbing continuous-time finite Markov chains. J. Appl. Prob. 4, 192-196.

[7] Ferrari, P. A., Kesten, H., Martinez, S. and Picco, P. (1995). Existence of quasistationary distributions. A renewal dynamical approach. Ann. Prob. 23, 501-521. 
[8] Keilson, J. and Ramaswamy, R. (1984). Convergence of quasi-stationary distributions in birth-death processes. Stoch. Process. Appl. 18, 301-312.

[9] Kryscio, R. J. and Lefèvre, C. (1989). On the extinction of the S-I-S stochastic logistic epidemic. J. Appl. Prob. 27, 685-694.

[10] Lefèvre, C. And Utev, S. (1995). Poisson approximation for the final state of a generalized epidemic process. Ann. Prob. 23, 1139-1162.

[11] Lefèvre, C. And Utev, S. (1997). Mixed Poisson approximation in the collective epidemic model. Stoch. Process. Appl. 69, 217-246.

[12] Matis, J. H. AND Kiffe, T. R. (1996). On approximating the moments of the equilibrium distribution of a stochastic logistic model. Biometrics 52, 980-991.

[13] NÅsELl, I. (2001). Extinction and quasi-stationarity in the Verhulst logistic model. J. Theoret. Biol. 211, 11-27.

[14] NÅsell, I. (2003). An extension of the moment closure method. Theoret. Pop. Biol. 64, 233-239.

[15] NÅselL, I. (2007). Extinction and quasi-stationarity in the Verhulst logistic model: with derivations of mathematical results. Available at http://www.math.kth.se/ ingemar/forsk/verhulst/ver10.pdf.

[16] NÅSELL, I. (2011). Extinction and Quasi-Stationarity in the Stochastic Logistic SIS Model. Springer, Heidelberg.

[17] NÅSELL, I. (2012). Extinction and quasi-stationarity in the stochastic logistic SIS model: a Maple module and corrections. Available at http://www.math.kth.se/ ingemar/SIS/SIS.html.

[18] Norden, R. H. (1982). On the distribution of the time to extinction in the stochastic logistic population model. Adv. Appl. Prob. 14, 687-708.

[19] Ovaskainen, O. (2001). The quasistationary distribution of the stochastic logistic model. J. Appl. Prob. 38, 898-907.

[20] Pollett, P. K. and Vassallo, A. (1992). Diffusion approximations for some simple chemical reaction schemes. Adv. Appl. Prob. 24, 875-893.

[21] Weiss, G. H. And Dishon, M. (1971). On the asymptotic behaviour of the stochastic and deterministic models of an epidemic. Math. Biosci. 11, 261-265. 